\title{
FLORA OF TABLE CAPE
}

\author{
by J.H. Willis
}

(with two plates and an appendix)

Historical notes are provided on the original vegetation of Table Cape before its alteration by extensive agricultural clearing, as well as a description of the current vegetation. Floristic details of the four major current habitats are given. A census is presented of 248 species of vascular plants, bryophytes and lichens, including exotics, fecorded at the Cape beiween 1832 and 1967.

Key Words: flora, Table Cape, early vegetation, vascular plants, bryephytes, lichens, Tasmania.

In BANKS, M.R. et al. (Eds), 1991 (31:iii): ASPECTS OF TASMANIAN BOTANY - A TRIBUTE TO WINIFRED CURTIS. Roy. Soc. Tasm. Hobart: 173-178. https://doi.org/10.26749/rstpp.124.2.173

\section{INTRODUCTION}

The northwestern coast of Tasmania, between Burnie and Robbins Island, is interrupted by three bold equidistant bluffs - Table Cape near Wynyard, Rocky Cape, and Circular Head (or The Nut) at Stanley; all were given these names by Captain Matthew Flinders on 5 December 1798 (Flinders 1814). Table Cape and The Nut have much in common, both consisting of basalt, rising about $150 \mathrm{~m}$ above the sea and formerly carrying lofty eucalypt forest. Intervening Rocky Cape is quite different - white quartzite of Precambrian origin, with a predominantly heathland flora rich in species (at least 260 indigenous kinds, including 46 orchids). Floristic accounts of the vegetation on The Nut and Rocky Cape were published by Willis (1966) and Firth (1969) respectively. It is, thus, appropriate that some record be made of the plants, both native and naturalised, known to inhabit the Table Cape promontory.

\section{HISTORICAL NOTES}

Settlement at Table Cape, with the consequent impact on the pristine vegetation, did not commence until the early 1850's - some 25 years after Van Diemens Land Company settlement at and around The Nut in 1826. Visiting missionary-botanist James Backhouse (1843) had given the first description of the Cape in its natural condition. Coming from Circular Head, doubtless along the VDL Company's rough bush track, he ascended the summit plateau on 14 November 1832 and was impressed by the "luxuriant vegetation" which included "Stately Stringybarks and White Gums, attaining to about 200 feet in height"; he specifically mentioned tree ferns (Dicksonia antarctica), prickly acacia (Acacia verticillata), musky aster (Olearia argophylla - pl. 1), Macquarie Harbour vine (Muehlenbeckia adpressa) and white clematis (Clematis aristata).

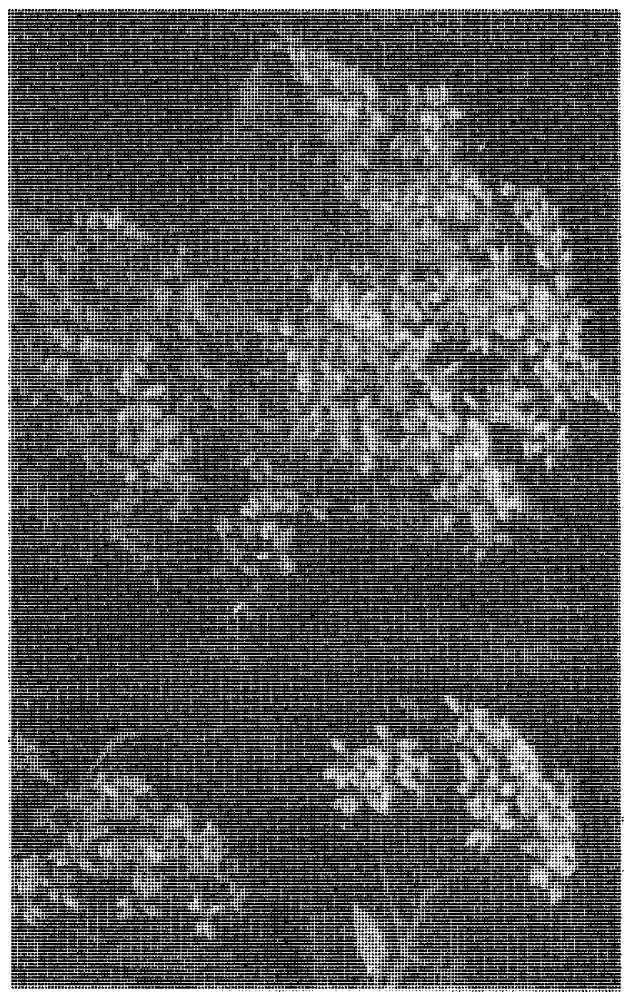

\section{PLATE I}

Musk daisy-bush (Olearia argophylla), now extremely rare at Table Cape. (Photo: Fred Bishop.)

Mercer (1963-64) described the activitics of pioneer settlers in the area. By 1850, surveyor Peter Lemonde Lette was occupying one of the first houses in what is now Wynyard, on the east bank of the Inglis River. From this base, Lette surveyed Table Cape, dividing the cape into 50 acre (approx. 20 ha) holdings .- with 
the exception of some Crown land reserved for a lighthouse, which was not completed until 1888. A major pioneer settler on the subdivided land of the Cape was George Shekleton from Dundalk, Ireland, who, in 1853, selected 1000 acres (405 ha) (later increased to 2000 acres $(810$ ha) $)$; he built Tollymore Cottage and resided there until his death in 1876 . Anorher small settlement had been established about 1851 by the Alexander brothers, near the present Table Cape road bridge.

Before the turn of the century, most of the densely forested country on Table Cape, that had so impressed Backhouse in 1832, had been replaced by open farmland on the rich, red volcanic soil. Some forest has persisted on the western slopes until today, viz. Dakings Bush (pl. 2), and contains a number of plants, e.g. the leguminous Pultenaea juniperina, Goodia lotifolia, Indigofera australis and Glycine clandestina, the lily Arthropodium milleflorum and two orchids, Pterostylis nutans and $P$. pedunculata, that are now absent from the contrastingly denuded surface of The Nut at Stanley.

Spicer (1878) frequently cited Circular Head as a locality for species, Rocky Cape occasionally (notably for orchids), but Table Cape not at all. His records are undoubtedly based largely on the collections of Ronald C. Gunn, Tasmania's greatest amateur botanist who was Police Magistrate at Circular Head from 1836-38. Rodway (1903) echoed all the records of Spicer for Circular Head and Rocky Cape, but ignored Table Cape - with one exception, the rare slender tree fern (Cyathea cunninghamii), which has long since been presumed extinct hereabouts. He also (Rodway 1913, 1914) recorded seven species of mosses from Circular Head and an eighth from Table Cape, Sphagnum brotherusii, which is probably referable to the common S. cynbifolioides C. Muell.

During January 1967, while the author was staying at Wynyard, he took the opportunity to make a list of the plant species on Table Cape. Only two days could be spared for this project, and the census in the appendix is a result of that effort. This list is not intended to be comprehensive; further critical examinations throughout every season are bound to augment it considerably.

\section{PRINCIPAL PLANT HABITATS}

\section{Open Eucalypt Forest}

Reference has been made to surviving tree cover (Dakings Bush) on the western slopes. Eucalyptus viminalis and E. obliqua are the dominamt species here, with Pomaderris apetala, Acacia melanoxylon and $A$. verticillata among subordinate trees or tall shrubs; a sparse herbaceous ground flora includes Acaena novae-zelandiae, Poranthera microphylla, Stackhousia monogyna, Hypericum gramineum, Viola hederacea and Gonocaipus teucrioides.

\section{Paperbark Thicket}

A large portion of the Table Cape plateau originally carried a dense growth of tall Melaleuca ericifolia over all persistent swamplands or areas with high water table. Sizeable relics of this formation still exist south from the present lighthouse, draining into a creek that forms a small waterfall over the northeastern escarpment. Most of the recorded ferri species, sedges, such taller shrubs as Pittosporum bicolor, Tasmannia lanceolata and Zieria arborescens are to be found here, with a few shade-tolerant herbs.

\section{Tussock Grassland and Herbfield (Induced)}

As on The Nut, areas cleared for pasture and crops have largely evolved into open grasslands dominated by tussock-forming perennials (e.g. introduced Dactylis glomerata (cocksfoot) and Lolium perenne (perennial rye-grass)), coexisting in mixture with the indigenous Danthonia species (wallaby grasses) and, in damper places, Poa labillardieri (tussock grass). Cropland may become a herbfield presenting a plethora of alien plants - clovers (Trifolium), medics (Medicago), vetches (Vicia), chickweeds (Stellaria and Cerastium), thistles (Carduus, Cirsium and Sonchus) etc. Grasses, with at least 33 species, account for $17 \%$ of the total vascular flora.

\section{Coastal Declivities and Cliffs}

The steepest terrain on Table Cape, west, north and east of the lighthouse at about $120 \mathrm{~m}$ a.s.l., affords the most diverse and complex of habitats - everything from a community of stunted trees (e.g. Banksia marginata, Exocarpos cupressiformis, Bursaria spinosa and Leucopogon parviflorus) at the edge of escarpments to a selvage of salt-tolerant plants against the sea — notably Stipa stipoides (coast spear grass), Distichlis distichophylla (salt grass), Sarcocornia quinqueflora (beaded glasswort), Carpobrotus rossii (karkalla or noon-flower), Muehlenbeckia adpressa (Macquarie vine or Macquarie grape), Apium prostratum (sea celery), Samolus repens (brookweed), Lobelia alata (angled lobelia) and small tree Myoporum insulare (common boobialla). Shrubs of the escarpments include Correa alba (white correa), Cyathodes juniperina (crimson berry), Alyxia buxifolia (sea box) and Solanum vescum (kangaroo apple). Rock crevices are colonised by Asplenium flabellifolium (necklace fern), Pleurosorus rutifolius (blanket fern), Einadia nutans (nodding saltbush), Pelargonium australe (austral stork's bill), Galium australe (tangled bedstraw) etc. 


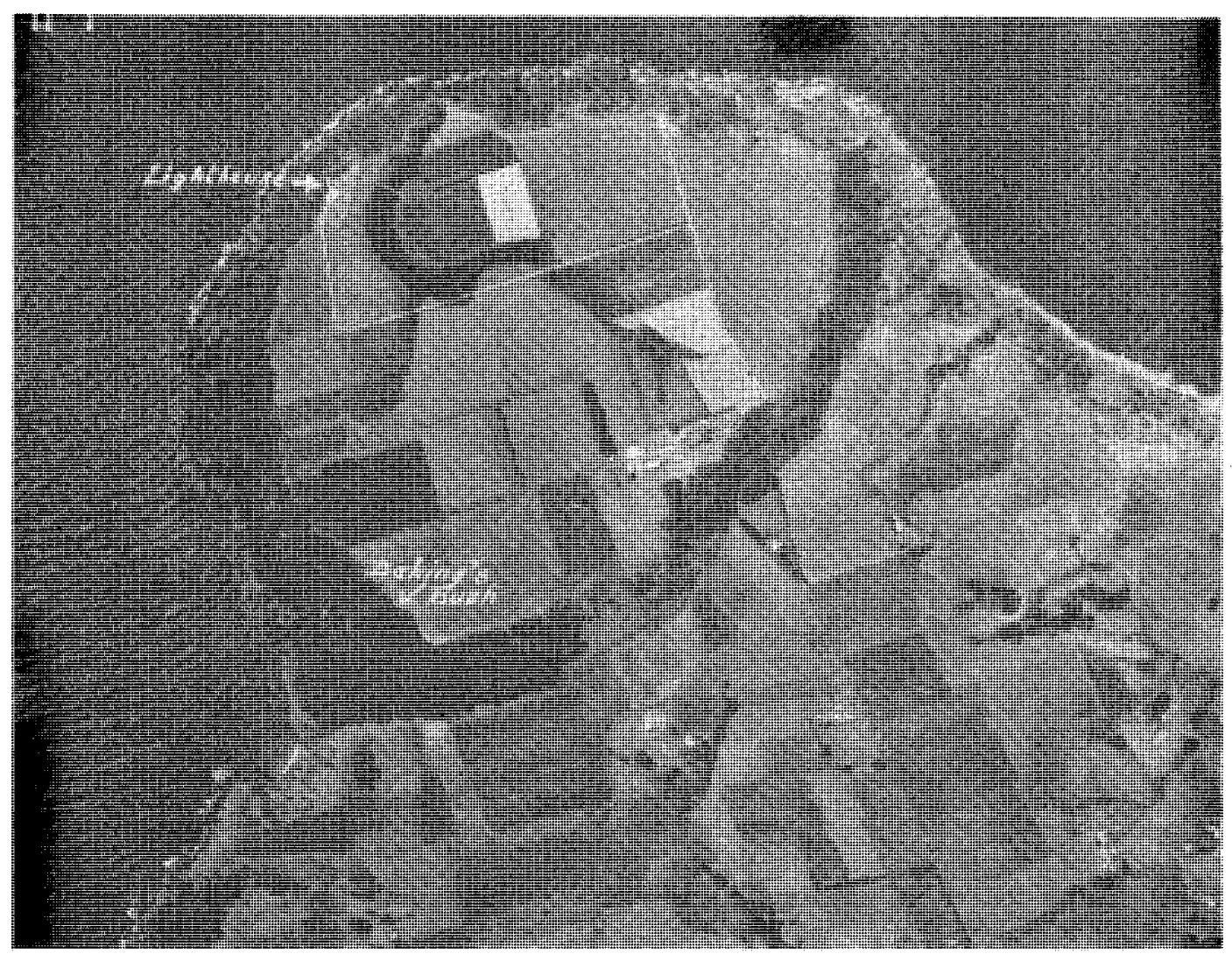

PLATE 2

Aerial view of Table Cape. (Photo: Dept of Planning and Environment, Hobart.)

\section{CENSUS OF PLANT SPECIES}

Of the 190 species of vascular plants listed in the appendix, 63 are naturalised aliens. Their arrangement follows that of Buchanan et al. (1989), wherein families, genera and species are all set out alphabetically under each of the major headings Pteridophytes (ferns), Monocotyledons and Dicotyledons. Bryophytes (mosses and hepatics - 41 species) and a few lichens are listed separately and arranged alphabetically as to genera and species, familial names being excluded.

\section{ACKNOWLEDGEMENTS}

The author is grateful for assistance from Mr Charles Turner, Burnie, who kindly supplied material from articles by Peter Mercer in the Arts Council Bulletin (November 1963 and July 1964); the late Mr M.J. Firth, who recorded the two greenhood orchids from Dakings Bush; the library of the Royal Botanic Gardens and National Herbarium, Melbourne; and Tasmap Sales Centre, Hobart, which provided the acrial photograph of Table Cape. 


\section{REFERENCES}

BACKHOUSE, J., 1843: A NARRATIVE OF A VISIT TO THE AUSTRALIAN COLONIES: 110-111.

Buchanan, A.M., McGeary-Brown, A. \& Orchard, A.E., 1989: A CENSUS OF THE VASCULAR PLANTS OF TASMANIA.Tasm. Herb. Occ Publ. No. 2:82 pp.

Firth, M.J., 1969: Flora of Rocky Cape. Rec. Q. Vict. Mus. 33: $1-16$.

FLINDERs, M., 1814: A VOYAGE TO TERRA AUSTRALIS I. G. \& W. Nicol, London: clxvi-vii.

Mercek, P., 1963 64: The early days of the Far North West Coast. Parts XIV-XX. Arts Council Bull. Nov. 1963 and Jul. 1964.
RodWay, L., 1903: THE TASMANIAN FLORA. Govt Printer, Hobart.

Rodway, L., 1913: Tasmanian Bryophyta, Parts 1 and 2. Pap. Proc. R. Soc. Tasm. 1912: 3-24, 87-138.

Rodway, L., 1914: Tasmanian Bryophyta, Part 3. Pap. Proc. R. Soc. Tasm. 1913: 177-263.

SPICER, W.W., 1878: A HANDBOOK OF THE PLANTS OF TASMANIA. J. Walch \& Sons, Hobart.

Wilurs, J.H., 1966: Flora of The Nut. Rec. Q. Vict. Mus. 21: $1-12$.

(accepted 14 March 1990)

J.H. Willis

102 Male Street, Brighton, Victoria, Australia 3186

\section{APPENDIX \\ Census of Plant Species on Table Cape}

An asterisk $(*)$ indicates naturalised aliens, $(\dagger)$ denotes a species now restricted to a large paperbark swamp south of the lighthouse, $(+)$ signifies any species that occurs also on The Nut.

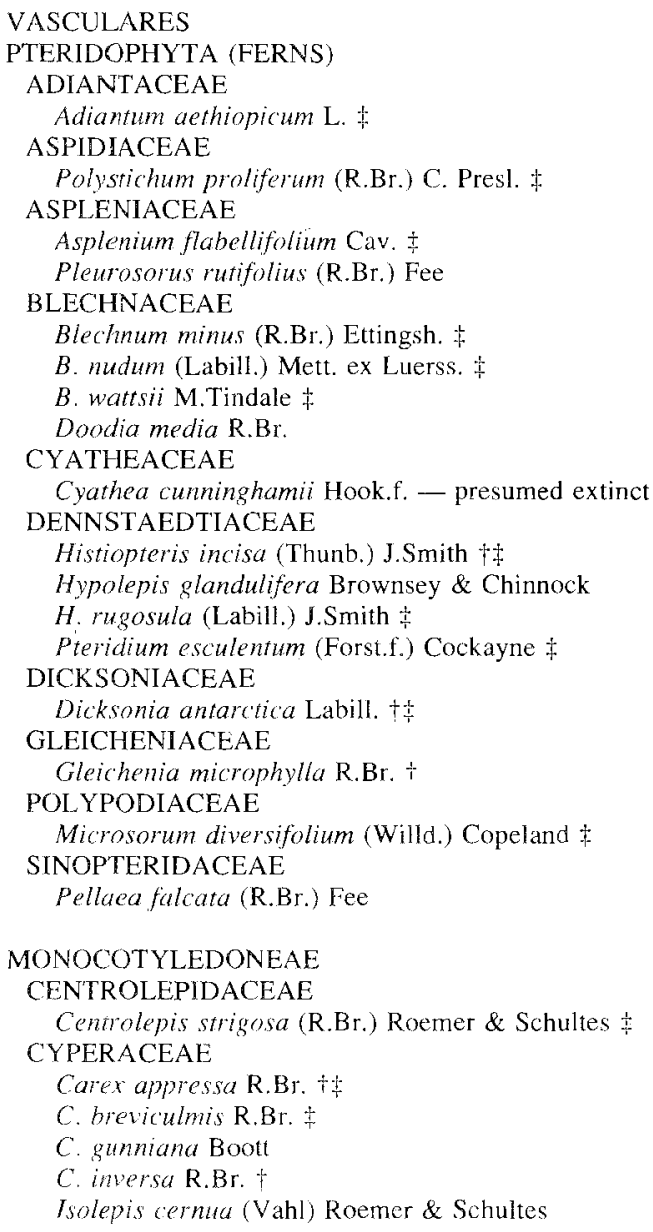

1. inundata $\mathrm{R} . \mathrm{Br} .+$

I. nodosa (Rottb.) R.Br. $\neq$

I. subtillissima Boeckler $\dagger$

Lepidopserma gladiatum Labill. $\ddagger$

L. laterale var. majus Benth. $¥ \ddagger$

IRIDACEAE

Diplarrena moraea Labill. $\ddagger$

JUNCACEAE

Juncus bufonius L. \$

J. ?effusus L. *†

J. kraussii Hochst.

J. pallidus R.Br. $\ddagger$

$J$ pauciflorus $\mathrm{R} . \mathrm{Br} . \dagger$

Luzula meridionalis Nordensk.

L. flaccida (Buchenau) Edgar

LILIACEAE

Arthropodium milleflorum (DC.) Macbride

Bulbine bulbosa (R.Br.) Haw.

Dianella revoluta $\mathrm{R} . \mathrm{Br} .+$

D. tasmanica Hook.f.

ORCHIDACEAE

Pterostylis nutans R.Br. Rock ledges in Dakings Bush.

P. pedunculata R.Br. Rock ledges in Dakings Bush. POACEAE

Agrostis avenacea J.Gmelin

A. capillaris L. $* \frac{4}{4}$

A. aff. hiemalis (Walt.) Britton et al.

Aira caryophyllea L. ${ }^{*}+$

Avena fatua L. ${ }^{*} \ddagger$

Briza minor L. *

Bromus diandrus Roth. * +

$B$. hordeaceus $*+$

$B$. sterilis L. *

Cynosurus cristatus L. *

C. echinatus L.*

Dactylis glomerata L. *

Danthonia caespitosa Gaudich.

D. pilosa $\mathrm{R} . \mathrm{Br}$. 
D. racemosa $\mathrm{R} . \mathrm{Br}$.

Deyeuxia quadriseta (Labill.) Benth.

Dichelachne crinita (L.f.) Hook.f.

Distichlis distichophylla (Labill.) Fassett

Echinopogon ovatus (Forst.f.) P.Beauv.

Elymus scaber (R.Br.) A.Löve $\ddagger$

Ehrharta stipoides Labill. $\ddagger$

Glyceria maxima (Hartman) Holmb. *

Holcus lanatus L. *末.

Hordeum murinum ssp. leporinum (Link) Arcang. *

Lolium perenne L. *\$

Phalaris minor Retz.*

Poa annua L. *

$P$. labillardieri Steudel $\ddagger$

$P$. poiformis (Labill.) Druce $\ddagger$

$P$. tenera F.Muell. ex Hook.f.

Stipa stipoides (Hook.f.) Veldk. 末

Vulpia bromoides (L.) Gray * $\ddagger$

XANTHORRHOEACEAE

Lomandra longifolia Labill. $\ddagger$

\section{DICOTYLEDONEAE}

AIZOACEAE

Carpobrotus rossii (Haw.) Schwantes $\ddagger$

Tetragonia implexicoma (Miq.) Hook.f. $\ddagger$

T. tetragonioides (Pallas) Kuntze

APIACEAE

Apium prostratum Labill. ex Vent. $\$$

APOCYNACEAE

Alyxia buxifolia R.Br. $\ddagger$

Vinca major L. *

ASTERACEAE

Actites megalocarpa (Hook.f.) N.Lander $\$$

Bellis perennis L. *

Carduus tenuiflorus Curtis *

Cassinia aculeata (Labill.) R.Br.

Cirsium vulgare (Savi) Ten. * ${ }^{*}$

Gnaphalium involucratum Forst.f. $\ddagger$

Hypochoeris radicata *末

Olearia argophylla (Labill.) Benth. $\ddagger$

O. lirata (Sims.) Hutch.

O. ramulosa (Labill.) Benth.

Picris hieracioides L. *

Senecio hispidulus A.Rich. $\neq$

$S$. lautus Forst.f. ex Willd. $\neq$

S. linearifolius A.Rich. $\ddagger$

S. minimus Poiret $\dagger$

Sonchus asper (L.) Hill. *

S. oleraceus *

BRASSICACEAE

Brassica rapa $\mathrm{L}$. *

Capsella bursa-postoris (L.) Medikus *

Rorippa nasturtium-aquaticum (L.) Hayek *†末

Sisymbrium officinale (L.) Scop. *

CAMPANULACEAE

Lobelia alata Labill. $\ddagger$

Wahlenbergia gracilis (Forst.f.) Schrader $\ddagger$

CAPRIFOLIACEAE

Sambucus gaudichaudiana DC. $\dagger+$

S. nigra L. *

CARYOPHYLLACEAE

Cerastium glomeratum Thuill. * +
Sagina procumbens L. * $\ddagger$

Silene gallica L. * $+\frac{+}{+}$

Stellaria media (L.) Cyrillo* $*$

S. pungens Brongn.

CHENOPODIACEAE

Atriplex prostrata Boucher ex DC. ${ }^{*+}+$

Chenopodium album $\mathrm{L}$. *

Einadia nutans (R.Br.) A.J. Scott

Rhagodia candolleana Moq. 末

Sarcocornia quinqueflora (Bunge ex Ung.-Sternb.) A.J. Scott $\ddagger$

CLUSIACEAE

Hypericum gramineum Forst.f.

CONVOLVULACEAE

Dichondra repens J.R. \& Forst.f. \$

CRASSULACEAE

Crassula sieberiana (Schultes \& Schultes) Druce $\ddagger$

EPACRIDACEAE

Cyathodes juniperina (Forst.) Druce $\ddagger$

Leucopogon ericoides (Smith) R.Br.

L. parviflorus (Andr.) Lindl. $\ddagger$

EUPHORBIACEAE

Poranthera microphylla Brongn. $\ddagger$

FABACEAE

Acacia melanoxylon R.Br. in Aiton $\ddagger$

A. verticillata (L'Herit.) Willd. $\ddagger$

Genista monspessulana (L.) L.Johnson *

Glycine clandestina J.Wendl.

Goodia lotifolia Salisb.

Indigofera australis Willd.

Medicago polymorpha $\mathrm{L} . *+$

Pultenaea daphnoides J.Wendi. $\ddagger$

$P$. juniperina Labill.

Trifolium dubium Sibth. * $\ddagger$

$T$. pratense $\mathrm{L}$. *

T. repens $\mathrm{L} .{ }^{*}+$

T. subterraneum L. *

Vicia sativa $\mathrm{L} . * \frac{*}{4}$

V. tetrasperma (L.) Schreber $* \frac{1}{+}$

FUMARIACEAE

Fumaria officinalis L. *

GERANIACEAE

Erodium moschatum (L.) L'Herit. ex Aiton *

Geranium solanderi Carolin $\ddagger$

Pelargonium australe Willd. $\ddagger$

HALORAGACEAE

Gonocarpus teucrioides DC.

LAMIACEAE

Prunella vulgaris $\mathrm{L}$.

LINACEAE

Linum marginale A.Cunn. ex Planchon

MYOPORACEAE

Myoporum insulare R.Br. $\ddagger$

MYRTACEAE

Eucalyptus obliqua L'Herit.

E. viminalis Labill. $\ddagger$

Melaleuca ericifolia Smith $\ddagger$

ONAGRACEAE

Epilobium billardierianum Ser. ex DC. +

OXALIDACEAE

Oxalis latifolia Humb. et al. *

O. perennans Haw. $\ddagger$ 


\section{PITTOSPORACEAE}

Bursaria spinosa var. macrophylla Hook. $\ddagger$ Pittosporum bicolor Hook. $\dagger$

PLANTAGINACEAE

Plantago lanceolata L. *

$P$. varia R.Br.

POLYGONACEAE

Muehlenbeckia adpressa (Labill.) Meissner

Rumex acetosella $\mathrm{L}$. * $\dagger$

$R$. brownii Campdera

$R$. crispus L. *ț

R. pulcher $\mathrm{L} . * \ddagger$

PORTULACACEAE

Calandrinia calyptrata Hook.f. $\ddagger$

C. caulescens Humb. et al. *

PRIMULACEAE

Anagallis arvensis $\mathbf{L} .{ }^{*} \ddagger$

PROTEACEAE

Banksia marginata Cav. $\ddagger$

RANUNCULACEAE

Clematis aristata R.Br. ex DC.

Ranunculus repens $\mathrm{L}$. *

RHAMNACEAE

Pomaderris apetala Labill. $\ddagger$

ROSACEAE

Acaena novae-zelandiae Kirk. $\ddagger$

A. echinata Nees in Lehm. $\ddagger$

Crataegus monogyna Jacq. $*$

Rosa rubiginosa L. *

Rubus fruticosus agg. *

$R$. parvifolius $\mathrm{L} . *+$

RUBIACEAE

Coprosma quadrifida (Labill.) Robinson $\nmid$ 拉

Galium aparine L. *末

G. australe DC. $\div$

G. gaudichaudii DC. $\ddagger$

Sherardia arvensis $\mathrm{L},{ }^{*}+$

RUTACEAE

Correa alba Andrews ‡

Zieria arborescens Sims $\dagger$

SANTALACEAE

Exocarpos cupressiformis Labill.

SAPINDACEAE

Dodonaea viscosa ssp. spathulata (Smith) J.West

SCROPHULARIACEAE

Parentucellia viscosa (L.) Caruel *

Verbascum thapsus L. *

Veronica arvensis L. *

SOLANACEAE

Solanum nigrum L. *

S. vescum F.Mucll.

STACKHOUSIACEAE

Stackhousia monogyna Labill.

URTICACEAE

Urtica incisa Poiret + t:

VIOLACEAE

Viola hederacea Labill. $\ddagger$

WINTERACEAE

Tasmannia lanceolata (Poiret) A.C.Smith $\dagger$
BRYOPHYTA

MUSCI (MOSSES)

Achrophyllum dentatum (Hook.f. \& Wils.) Vitt \& Crosby

Amphidium cyathicarpum (Mont.) Broth.

Barbula calycina Schwaegr.

Bartramia ithyphylla Brid.

Breutelia affinis (Hook.) Mitt.

Bryum billardieri Schwaegr.

B. clavatum (Schimp.) C.Muell.

B. subapiculatum Hampe

Calyptrochaeta apiculata (Hook.f. \& Wils.) Vitt

Camptochaete gracilis (Hook.f. \& Wils.) Par.

Campylopus bicolor (Hornsch. ex C.Muell.) Wils.

C. introflexus (Hedw.) Brid.

Ceratodon purpureus (Hedw.) Brid.

Ditrichum cylindricarpum (C.Muell.) F.Muell.

Fissidens asplenioides Hedw.

$F$. leptocladus C.Muell. ex Rodw.

$F$. pallidus Hook.f. \& Wils.

$F$. tenellus Hook.f. \& Wils.

Funaria hygrometrica Hedw.

Grimmia pulvinata (Hedw.) Sm.

Hypnum cupressiforme Hedw.

Lembophyllum divulsum (Hook.f. \& Wils.) Lindb.

Philonotis tenuis (Tayl.) Reichdt.

Racomitrium crispulum (Hook.f. \& Wils.) Hook.f. \& Wils.

Racopilum convolutaceum (C.Muell.) Reichdt.

Rhynchostegium tenuifolium (Hedw.) Reichdt.

Sematophyllum homomallum (Hampe) Broth.

Sphagnum cymbifolioides C.Muell.

Thuidium furfurosum (Hook.f. \& Wils.) Reichdt.

Tortula papillosa Wils.

T. princeps De Not.

Weissia controversa Hedw.

Zygodon intermedius B.S.G.

HEPATICAE (LIVERWORTS)

Chiloscyphus biciliatus (Hook.f. \& Tayl.) Gott. et al.

C. muricatus (Lehm.) Engel \& Schust.

C. semiteres (Lehm.) Lehm. \& Lindenb.

Frullania falciloba Tayl. ex Lehm.

Lejeunea drummondii Tayl.

Metzgeria furcata (L.) Dumort.

Plagiochila fasciculata Lindenb.

Zoopsis leitgebiana (Carr. \& Pears.) Bastow

LICHENS

Caloplaca ? cinnabarina (Ach.) Zahlbr.

Cladia aggregata (Sw.) Nyl.

Flavoparmelia rutidota (Hook.f. \& Tayl.) Hale

Lecanora rupicola (L.) Zahlbr.

Lecidea sp.

Pseudocyphellaria crocata (L.) Vainio

Ramalina celastri (Sprengel) Krog \& Swinsc.

$R$. inflata (Hook.f. \& Tayl.) Hook.f. \& Tayl.

Rhizocarpon sp. [minute black apothecia]

Rinodina ?thiomela (Nyl.) Müll.Arg.

Stereocaulon ramulosum ( $\mathrm{Sw}$.) Räusch.

Sticta sp. [grey]

Teloschistes chrysophthalmus (L.) Th.Fr.

T. ?spinosus (Hook.f. \& Tayl.) J.Murray

Tephromela ?atra (Huds.) Hafelner

Xanthoparmelia ?tasmanica (Hook.f. \& Tayl.) Hale Xanthoria ligulata (Körber) P.James 